\title{
Understanding risk of PML through multiple sclerosis
}

\author{
Martin, Roland
}

DOI: https://doi.org/10.1016/s1474-4422(18)30122-4

Posted at the Zurich Open Repository and Archive, University of Zurich ZORA URL: https://doi.org/10.5167/uzh-158841

Journal Article

Accepted Version

Originally published at:

Martin, Roland (2018). Understanding risk of PML through multiple sclerosis. Lancet Neurology, 17(5):391-392. DOI: https://doi.org/10.1016/s1474-4422(18)30122-4 


\title{
Understanding risk of PML through multiple sclerosis
}

\author{
Roland Martin \\ Neuroimmunology and MS Research \\ Neurology Clinic \\ University Hospital Zurich \\ University Zurich \\ Frauenklinikstrasse 26 \\ 8091 Zurich \\ Switzerland
}

Progressive multifocal leukoencephalopathy, PML, an often fatal opportunistic infection caused by JC virus, which most human beings carry throughout life without consequences, was identified in $1959(1,2)$. After being a major cause of death in AIDS patients, its prevalence sharply declined with the introduction of retroviral therapy. It is now mostly seen in patients during chemotherapy and immunosuppression. The first PML cases in natalizumab-treated multiple sclerosis patients came as a surprise in $2005(4,5)$ since PML had not been observed with the multiple sclerosis medications interferon-beta (IFN-b) and glatiramer acetate (GA) before, and also since multiple sclerosis patients - except for an autoimmune reaction against central nervous system (CNS) tissue - are immunologically healthy, i.e. they cope well with infections and show no increased risk of malignancies. These cases therefore led the regulatory authorities to suspend approval, although natalizumab was allowed back on the market in 2006 after risk factors for PML were identified(6) and the manufacturer provided a reasonable path to manage the risks through the introduction of a sensitive assay to measure antibody responsiveness against JCV. In their Review,(3) Eugene Major and colleagues discuss how instructive PML as serious adverse event of natalizumab treatment in multiple sclerosis has been both with respect to a better understanding of PML pathogenesis, but importantly also for better patient management.

A puzzling observation is that natalizumab-treated patients with high anti-JCV antibody indices carry a high risk of developing PML (1/70 or higher), while risk is much lower in those with low antibody titers and negligible in anti-JCV negative individuals (6). Besides regular antibody testing, the routine performance of MRI to identify early imaging signs of incipient PML even prior to clinical manifestation is another important step in lowering the risk of PML or diagnosing it before serious brain damage has occurred. Major and colleagues provide guidance on how often patients should be scanned, which MRI findings hint at the beginning of PML, how to identify PML immune reconstitution inflammatory syndrome (IRIS) as a sign of an immune response against the infected brain cells versus expanding PML lesions, and which steps should be taken to manage the patient once MRI lesions that indicate PML occur. The authors discuss how to recognise PML early-by identifying increasing JCV-specific antibodies in both serum and CSF, compatible MRI lesions, and JC viral DNA in the CSF-alongside the limitations of current diagnostic methods, for example the paucity of longitudinal serological data and that negative JCV CSF viral load alone does not exclude PML. The authors also summarize the evolution of JCV-specific antibodies and DNA across the stages of PML in urine, blood and CSF, describe further the clinical stages of the disease, and give recommendations for PML surveillance.

However, due to scarcity of studies on PML, several aspects of the Review seem incomplete. For example, the monitoring recommendations, which are probably the most important 
aspect for treating neurologists, are too complicated and, given the severity of the disease, not cautious enough in minimizing PML risk. A much simpler scheme that we routinely use stratifies patients into those without antibodies (presumed JCV negative), those with an antibody index of $\leq 0.9$, and those with $>0.9$. In the first two strata, MRI scans and antibody index testing are performed every 6 months, and every 3 months in the high index patients. The MRI protocol is essentially the standard that is used for assessing disease activity of multiple sclerosis under treatment and includes pre- and post-contrast T1-, T2/FLAIR-, and diffusion-weighted sequences (DWI) once PML is suspected. Unless there are strong reasons or the patient wishes to continue after discussing the risks, natalizumab treatment is stopped after 1 year even in patients with indices $\leq 0.9$. Considering approximately 200 deaths from natalizumab-associated PML cases and overall more than 750 cases (7), many of these with severely disabling sequelae, and taking into account that highly effective alternatives to treat multiple sclerosis are available, this scheme appears justified. Neurologists in Sweden use an even simpler approach and only treat multiple sclerosis patients with natalizumab if they are JCV antibody negative. The fact that no PML case has been observed with natalizumab during the last 5 years in Sweden (T. Olsson, Karolinska Institute, personal communication) underscores that this position is the safest way to proceed.

Despite remaining questions, about the pathogenesis, diagnosis and treatment of PML the highly unexpected adverse event of natalizumab treatment in multiple sclerosis has urged physicians, scientists, and drug manufacturers to work together and find solutions to deal with this important issue. Through their efforts, PML is now much better understood, and even though it occurs only rarely with other multiple sclerosis drugs, it is a crucial topic to discuss with almost every newly diagnosed patient when considering treatment options. Yet, although therapeutic and prophylactic vaccination (8) and antiviral therapies are being explored and give hope that a treatment will be developed, the overall low number of PML cases and the lack of systematic reporting pose substantial hurdles.

1. Major EO. Progressive multifocal leukoencephalopathy in patients on immunomodulatory therapies. Annu. Rev. Med. 2010;61:35-47.

2. Tan CS, Koralnik IJ. Progressive multifocal leukoencephalopathy and other disorders caused by JC virus: clinical features and pathogenesis. Lancet Neurol. 2010 Apr;9(4):425-37

3. Major EO, Yousry TA, Clifford DB. Pathogenesis of progressive multifocal leukoencephalopathy and risks associated with treatments for multiple sclerosis: a decade of lessons learned. Lancet Neurol., ...

4. Van Assche G, Van Ranst M, Sciot R, et al. Progressive multifocal leukoencephalopathy after natalizumab therapy for Crohn's disease. New Engl. J. Med. 2005; 353: 362-8.

5. Langer-Gould A, Atlas SW, Bollen AW, Pelletier D. Progressive multifocal leukoencephalopathy in a patient treated with natalizumab. New Engl. J. Med. 2005; 353 : 375-81.

6. Plavina T, Subramanyam M, Bloomgren G, et al. Anti-JC virus antibody levels in serum or plasma further define risk of natalizumab-associated progressive multifocal leukoencephalopathy. Ann Neurol 2014; 76(6): 802-12.

7. Biogen. Medical information. Tysabri (natalizumab) safety update: December 2017. https://medinfo.biogen.com/secure/pmlresource 
8. Sospedra M, Schippling S, Yousef $S$, et al. Treating PML with interleukin-7 and vaccination with JC virus capsid protein VP1. Clin. Infect. Dis. 2014; 59 (11): 1588-92 